\title{
Radiation belt data assimilation of a moderate storm event using a magnetic field configuration from the physics-based RAM-SCB model
}

\author{
Y. Yu ${ }^{1}$, J. Koller ${ }^{1}$, V. K. Jordanova ${ }^{1}$, S. G. Zaharia ${ }^{1}$, and H. C. Godinez ${ }^{2}$ \\ ${ }^{1}$ Space Science and Application, Los Alamos National Laboratory, Los Alamos, NM, USA \\ ${ }^{2}$ Applied Mathematics and Plasma Physics, Los Alamos National Laboratory, Los Alamos, NM, USA \\ Correspondence to: Y. Yu (yiqun@lanl.gov)
}

Received: 7 December 2013 - Revised: 11 March 2014 - Accepted: 14 March 2014 - Published: 6 May 2014

\begin{abstract}
Data assimilation using Kalman filters provides an effective way of understanding both spatial and temporal variations in the outer electron radiation belt. Data assimilation is the combination of in situ observations and physical models, using appropriate error statistics to approximate the uncertainties in both the data and the model. The global magnetic field configuration is one essential element in determining the adiabatic invariants for the phase space density (PSD) data used for the radiation belt data assimilation. The lack of a suitable global magnetic field model with high accuracy is still a long-lasting problem. This paper employs a physics-based magnetic field configuration for the first time in a radiation belt data assimilation study for a moderate storm event on 19 December 2002. The magnetic field used in our study is the magnetically self-consistent inner magnetosphere model RAM-SCB, developed at Los Alamos National Laboratory (LANL). Furthermore, we apply a cubic spline interpolation method in converting the differential flux measurements within the energy spectrum, to obtain a more accurate PSD input for the data assimilation than the commonly used linear interpolation approach. Finally, the assimilation is done using an ensemble Kalman filter (EnKF), with a localized adaptive inflation (LAI) technique to appropriately account for model errors in the assimilation and improve the performance of the Kalman filter. The assimilative results are compared with results from another assimilation experiment using the Tsyganenko 2001S (T01S) magnetic field model, to examine the dependence on a magnetic field model. Results indicate that the data assimilations using different magnetic field models capture similar features in the radiation belt dynamics, including the temporal evolution of
\end{abstract}

the electron PSD during a storm and the location of the PSD peak. The assimilated solution predicts the energy differential flux to a relatively good degree when compared with independent LANL-GEO in situ observations. A closer examination suggests that for the chosen storm event, the assimilation using the RAM-SCB predicts a better flux at most energy levels during storm recovery phase but is slightly worse in the storm main phase than the assimilation using the T01S model.

Keywords. Magnetospheric physics (energetic particles, trapped)

\section{Introduction}

Data assimilation has recently become an increasingly important tool applied by the magnetospheric physics community for understanding the dynamics of the outer electron radiation belts. This technique provides an efficient way of combining sparse space-borne observations with physicsbased models and is capable of achieving a full spatial and temporal coverage. It can also compensate for missing physics in the models by providing corrections to the model output for a better forecast of the radiation belt dynamics (Koller et al., 2007). Earlier studies used "direct data insertion" as a primary tool in combining sparse, poorly distributed measurements in space with a physics-based radiation belt model to obtain a global representation of the radiation belt (e.g., Bourdarie et al., 2005). This technique assumes that the inserted observations are exact, which is however not true. 
The assimilation techniques involving a Kalman filter that takes into account uncertainties from both observations and the model prediction to provide an optimized estimation of the true state became more popular later on. Naehr and Toffoletto (2005) made an early effort of incorporating continuous observations and a physics-based model using an extended Kalman filter (EKF). This tool has been extensively applied to investigate complex loss and acceleration processes in the outer electron radiation belt. For instance, Kondrashov et al. (2007) included the model parameter estimation into the data assimilation process using the EKF, CRRES observations, and a radial transport code to characterize the lifetime of relativistic electrons and found that the assimilation process can effectively drive biased model parameters towards their true values. Shprits et al. (2012) carried out a statistical study via an EKF combining a radial diffusion model with measurements from multiple satellites to investigate the evolution of the location of local source and the peak of phase space density (PSD) in response to different geomagnetic activity levels. Recently, Daae et al. (2011) assessed the sensitivity of the data assimilation using the EKF to different boundary conditions, electron lifetimes, and initial conditions, and concluded that the dynamics of the heart of the radiation belt is independent on these model assumptions.

Another efficient assimilation tool is the ensemble Kalman filter (EnKF) first introduced by Evensen (2003). It employs a Monte Carlo approach in specifying uncertainties in both the model and data, and its main advantage is the ease of implementation and accuracy for assimilation in nonlinear models. Unlike the EKF, which linearizes the model dynamics to approximate the model error covariance matrix, the EnKF algorithm uses the Monte Carlo method in solving the error covariance with the full effect of nonlinear processes included. Application of this tool in the radiation belt has been demonstrated by Koller et al. (2007) to understand the acceleration mechanisms missing from the 1-D radiation belt model but being accounted for by the assimilation technique. (A version of EnKF and the radial diffusion radiation model are available with SpacePy (Morley et al., 2010; Welling et al., 2012).) Schiller et al. (2012) conducted a parametric study using the EnKF innovation vector to identify the source location and magnitude in the outer electron radiation belt. Recently, Bourdarie and Maget (2012) and Shprits et al. (2013) reported data assimilation in 3-D radiation belt models.

An important component in the Kalman filter is the error statistics, which are used to approximate the uncertainty in both the model and data. The uncertainty in the model comes from many sources, including incomplete physics, initial conditions, and approximate parameterizations. However, the model error is usually not adequately merged in the data assimilation, causing unreliable results. A recent study by Kondrashov et al. (2011) proposed a modification of Kalman filtering to use a log-transformed 1-D radiation belt model to reduce the model errors and found significantly improved analysis results. Alternatively, a new technique called localized adaptive inflation (LAI) was developed by Godinez and Koller (2012) to account for the underestimated model error, resulting in a well-reconstructed radiation belt electron PSD distribution. Unlike global inflation techniques, the LAI inflates the covariance matrix over a local domain, avoiding over-inflation and the introduction of noise into the assimilation solution. This is because the global inflation techniques either multiply a constant inflation factor to the error covariance matrix or apply an adaptively computed inflation factor to the global state vector. In this study we will use the LAI approach to better incorporate the model uncertainties into the ensemble Kalman filter in order to increase the effectiveness of data assimilation. Section 2.3 illustrates the assimilated PSD state using an EnKF with the LAI technique and illustrates its advantage over those with improper inflation.

To obtain the PSD data input for the radiation belt data assimilation, in situ differential flux measurements are used for conversion to PSD data. In this step, a global magnetic field model is a required element to calculate the adiabatic coordinates $\left(\mu, K, L^{*}\right)$ for the PSD data, because these coordinates are dependent on either the local or global magnetic field information. Here, $\mu$ is the magnetic moment or the first adiabatic invariant; $K$ is the modified longitudinal invariant depending on the magnetic field geometry; and $L^{*}$ is the Roederer $L$ shell (Roederer, 1970) associated with the third adiabatic invariant, i.e., the magnetic flux enclosed by the particle's guiding drift shell. At present, producing a global magnetic field model with high order of accuracy is still a demanding problem. Empirical models, such as the Tsyganenko 96 (T96) and Tsyganenko 2001S (T01S) models, postulate electric current systems with model parameters determined by fitting the resulting magnetic field to many observations. The T01S model is designed specifically for magnetic storms, whereas the T96 model is more general. These models have been widely used in the radiation belt data assimilation studies (e.g., Shprits et al., 2007; Daae et al., 2011; Koller et al., 2007), but their imperfection can pose considerable biases in the adiabatic invariants especially during highly disturbed time (e.g., Huang et al., 2008; Yu et al., $2012 \mathrm{~b}, 2014)$ and is expected to influence the subsequent assimilated PSD state.

On the other hand, physics-based magnetic field models have not yet been employed in the radiation belt data assimilation or evaluated in regards to their specific role in contrast to the empirical magnetic field models. Zaharia et al. (2006) and $\mathrm{Yu}$ et al. (2012a) reported that the magnetic field prediction from the ring current-atmosphere interaction model (RAM) self (S)-consistently (C) coupled with a 3-D magnetic field (B) code is generally more realistic than that from empirical models (e.g., T01S). Furthermore, the conversion of electron differential flux data to the PSD data requires interpolation techniques that could affect the accuracy of the input data in the data assimilation. Therefore, in order to achieve 
a more accurate assimilative result, we will use the above physics-based global magnetic field model combined with a more sophisticated interpolation technique to improve the inversion of observed differential flux into PSD, which will lead in turn to less biased data assimilation output. Finally, we perform another radiation belt data assimilation using the empirical magnetic field model T01S and compare both assimilation results to observations, to examine the dependence of the assimilative state on a magnetic field model. Ni et al. (2009) carried out a comparative study using various Tsyganenko empirical models (T89, T96, T01S, TS05) in the radiation belt data assimilation and found that the reanalysis results are relatively insensitive to the selected magnetic field model as the peak location of the electron PSD and the dynamic features are similarly produced. Here we step forward to assimilating the radiation belt with a physics-based magnetic field model. The comparison with results using empirical magnetic field models will help us identify in the global sense whether a physics-based magnetic field model represents the more realistic magnetospheric configuration.

\section{Methodology}

This section describes the three components in a radiation belt data assimilation study: the first-principle radiation belt model, PSD data, and the data assimilation algorithm. The PSD data are inverted from the observed differential flux data with the help of the RAM-SCB magnetic field configuration and a cubic spline interpolation method. The data assimilation algorithm consists of combining the EnKF with the LAI technique, a method recently developed in Godinez and Koller (2012), to better account for model errors within the radiation belt model. While the above LAI technique has already been developed and therefore adopted in this study, the goal here is to demonstrate the estimation of radiation environment from the data assimilation using physics-based magnetic fields.

\subsection{Radiation belt transport model}

The physical radiation belt model used in this study is a simplified one-dimensional Fokker-Planck equation:

$$
\frac{\partial f\left(L^{*}, t\right)}{\partial t}=L^{* 2} \frac{\partial}{\partial L^{*}}\left(\frac{D_{L L}}{L^{* 2}} \frac{\partial f\left(L^{*}, t\right)}{\partial L^{*}}\right)-\frac{f\left(L^{*}, t\right)}{\tau},
$$

where $f\left(L^{*}, t\right)$ is the electron radiation belt PSD at fixed ( $\mu$, $K$ ) coordinates; $D_{L L}$ is the radial diffusion coefficient from Brautigam and Albert (2000):

$$
D_{L L}\left(\mathrm{Kp}, L^{*}\right)=10^{(0.506 \mathrm{Kp}-9.325)} L^{* 10}, \mathrm{Kp} \in[1,6] .
$$

The electron lifetime $\tau$ is assumed to be 5 days inside the plasmapause and $5 / \mathrm{Kp}$ days outside the plasmapause, following Shprits et al. (2006). The plasmapause is determined by the empirical model from Carpenter and Anderson (1992) based on the Kp index. The outer boundary at $L^{*}$ of 10 is a free parameter that can be adjusted by the data assimilation result, and the inner boundary at $L^{*}=1.0$ is zero.

\subsection{Phase space density (PSD) data}

We obtain the PSD data, as a function of adiabatic coordinates $\left(\mu, K, L^{*}\right)$, by converting the in situ differential flux observations. Such a conversion invokes a global magnetospheric field model to yield both local and global magnetic fields for the computation of the adiabatic coordinates. While earlier researchers typically chose the Tsyganenko empirical magnetic field model for this procedure, this study uses a global magnetic field configuration generated from a physics-based inner magnetosphere model RAM-SCB.

\subsubsection{Physics-based magnetic field model RAM-SCB}

The magnetically self-consistent inner magnetosphere model RAM-SCB couples two codes: the kinetic ring currentatmosphere interactions model (RAM) (Jordanova et al., 1994, 2006, 2010) self-consistently (SC) coupled with a 3-D equilibrium magnetic field (B) code (Zaharia et al., 2004, 2006; Zaharia, 2008). This coupled model was originally confined within geosynchronous orbit and has been validated against a variety of in situ spacecraft observations and ground-based measurements in Yu et al. (2012a), which found reasonably good agreements between simulation results and observed magnetic field, ion differential flux, and geomagnetic index during storm times. The RAM-SCB model has now been expanded from the geosynchronous orbit to $9.0 R_{\mathrm{E}}$ (Jordanova et al., 2014). The RAM code evaluates the bounce-averaged distribution function for the ring current ions and electrons in the magnetic equatorial plane as a function of radial distance from the Earth (from 2 to $9.0 R_{\mathrm{E}}$ ), all magnetic local times, kinetic energy from 0.1 to $400 \mathrm{keV}$, and equatorial pitch angle $\alpha$ from $0^{\circ}$ to $90^{\circ}$. Loss processes, such as collisions with neutral and charged particles, and scattering by plasma waves, are included. The 3-D magnetic field code solves a plasma-force-balanced equation in flux coordinates (Euler potentials) with boundary conditions specified by output from the T89 empirical magnetic field model (Tsyganenko, 1989). The plasma anisotropic pressure produced from the ring current particle distribution function is passed to the 3-D magnetic field code, which in turn provides the magnetic field information to RAM to drift the ring current particles. The plasma boundary conditions at $9.0 R_{\mathrm{E}}$ are specified by an empirical solar-wind-driven plasmasheet model (Tsyganenko and Mukai, 2003). The convective electric field used for the particle transport and acceleration is specified by the Weimer electric potential model (Weimer, 2001).

The coupled model produces a global magnetic field configuration within $9.0 R_{\mathrm{E}}$, which is sufficient for calculating the adiabatic coordinates for particles drifting near the 
geosynchronous orbit during a moderate storm event. We use LANL-GEO satellites and Polar spacecraft to provide electron PSD data for the data assimilation.

\subsubsection{Flux-to-PSD conversion}

The PSD conversion from in situ observed differential flux data mainly follows the procedure described in Chen et al. (2005), which is briefly summarized here. At one location where the flux measurement is taken, given a set of $\left(\alpha_{0}\right.$, $\left.\mu_{0}\right)$ grids, the energy $E_{0}$ can be obtained via the conservation of the first adiabatic invariant. Meanwhile, the modified invariant $K$ is computed through the integral of $K=$ $\int_{s_{m 2}}^{s_{m 1}} \sqrt{B_{m}-B(s)} \mathrm{d} s$, and the $L^{*}$ is calculated from a global integration of encompassed magnetic flux inside the drift shell $L^{*}=2 \pi M /|\Phi| R_{\mathrm{E}}$ following the methodology outlined in Roederer (1970). The observed flux data $j$, which is a function of local pitch angle $\alpha$ and energy $E=\sqrt{E_{1} E_{2}}$ (where $E_{1}$ and $E_{2}$ represent the upper and lower bound of the energy channel), is then interpolated into the prescribed $\alpha_{0}$ grid. The interpolated flux $j\left(\alpha_{0}, E\right)$ is subsequently used to convert to PSD $f\left(\alpha_{0}, E\right)$ based on the following relation (Chen et al., 2005):

$$
\begin{aligned}
& f\left(\alpha_{0}, E\right)=\frac{j\left(\alpha_{0}, E\right)}{p^{2} c^{2}} \times 1.66 \times 10^{-10} \times 200.3, \\
& p^{2} c^{2}=E\left(E+2 E_{\mathrm{r}}\right),
\end{aligned}
$$

where $E_{\mathrm{r}}$ is the rest energy of the electron, the flux $j$ is in units of $\left(\mathrm{cm}^{2} \mathrm{srskeV}\right)^{-1}$, and the PSD $f$ is in units of $\mathrm{c}^{3} \mathrm{MeV}^{-3} \mathrm{~cm}^{-3}$. In order to find the PSD at $E_{0}$, an interpolation technique is required. Instead of fitting an exponential energy spectrum and then linearly interpolating between two neighboring energies (Ni et al., 2009; Chen et al., 2005), we alternatively use a cubic spline interpolation method. No extrapolation is allowed in this step even though only partial energy coverage of $E_{0}$ grids may be available from the spacecraft. The interpolated PSD $f\left(\alpha_{0}, E_{0}\right)$ is equivalent to $f\left(\mu_{0}\right.$, $\left.K, L^{*}\right)$. Finally, for a prescribed $K_{0}$ value, we again employ the cubic spline interpolation to procure the PSD $f\left(\mu_{0}, K_{0}\right.$, $\left.L^{*}\right)$, which is used as data input in the data assimilation. The above steps employ the cubic spline interpolation technique in two places: interpolation on the energy grids and interpolation on the $K$ grids. The reason to use a cubic spline interpolation method is because the standard linear interpolation results in systematic offset (either overestimate or underestimate depending on the energy spectrum) in the computed PSD values, as demonstrated below.

As the radiation belt data assimilation is largely dependent on the properties of the PSD data input (including its location and magnitude) for an optimal representation of the radiation environment, the accuracy of the PSD converted from the flux data is critically important. We carry out a reverse conversion from the transformed PSD $f$ to flux $j^{\prime}$ to evaluate the accuracy of the above conversion, especially in those steps involved with interpolation. It is expected that a perfect flux-to-PSD conversion should lead to a perfect agreement between the reversely converted flux $j^{\prime}$ and the original flux $j$. Figure 1a illustrates the agreement between the original flux data $j$ (black) and the reversely converted flux $j_{\text {cub }}^{\prime}$ (red) from PSD using a cubic spline interpolation technique as well as the converted flux $j_{\text {lin }}^{\prime}$ (blue) using a linear interpolation method. The offset in the flux $j_{\text {lin }}^{\prime}$ demonstrates that a bias develops in this interpolation, hence systematically misrepresenting the PSD data. A systematic offset in the PSD data can eventually incur a biased estimate of the radiation environment. This however can be prevented by the cubic spline interpolation approach, which is able to very well exhibit the relationship between the flux $j$ and PSD $f$ as the reversely converted flux $j_{\text {cub }}^{\prime}$ agrees very well with the original flux $j$. This interpolation method therefore helps eliminate unnecessary systematic errors in the data conversion step.

To understand the bias produced from the linear interpolation method, Fig. 1b illustrates the interpolated PSD values (blue triangles or red stars) over part of an energy spectrum from the original PSD data (black dots). The reverse conversion will use data points at the blue triangles or red stars to obtain PSD at point 1 or 2 . With a linear interpolation (using the dashed line), the interpolated value will appear above the original black dot at point 1 when the local spectrum has a concave shape and will appear below the original data at point 2 when the spectrum has a convex shape. Since the energy spectrum never appears as a straight line, the linear interpolation will inevitably introduce the offset when comparing with the original data points. However, applying the cubic spline interpolation method (following the red line), the bias can be minimized because the reversed conversion of PSD at point 1 and 2 will appear nearly at the original data point. After this step, the remaining uncertainty introduced to the $L^{*}$ calculation and the PSD conversion is due to the imperfection of the magnetic field configuration in a magnetic field model.

Before the PSD data are finally incorporated into the data assimilation, the inter-satellite calibration is necessary if multiple satellites are chosen to supply the PSD data for the data assimilation (Chen et al., 2005). This will remove the bias and inconsistency between the satellites and avoid an inherited bias in the data assimilation result. In this study, we use three LANL-GEO satellites and conduct a fine-tuned inter-calibration similar to Chen et al. (2005) and Ni et al. $(2009,2011)$ based on the Liouville theorem.

\subsection{Ensemble Kalman filter with localized adaptive inflation (LAI)}

The one-dimensional radiation belt model utilized in this work contains no source term. This missing physics as well as other approximation in the loss and transport processes can introduce model errors, which are usually not well specified in the 1-D radiation belt data assimilation. As a consequence, this can result in filter divergence, where the Kalman filter fails to converge towards the observations (Godinez and 


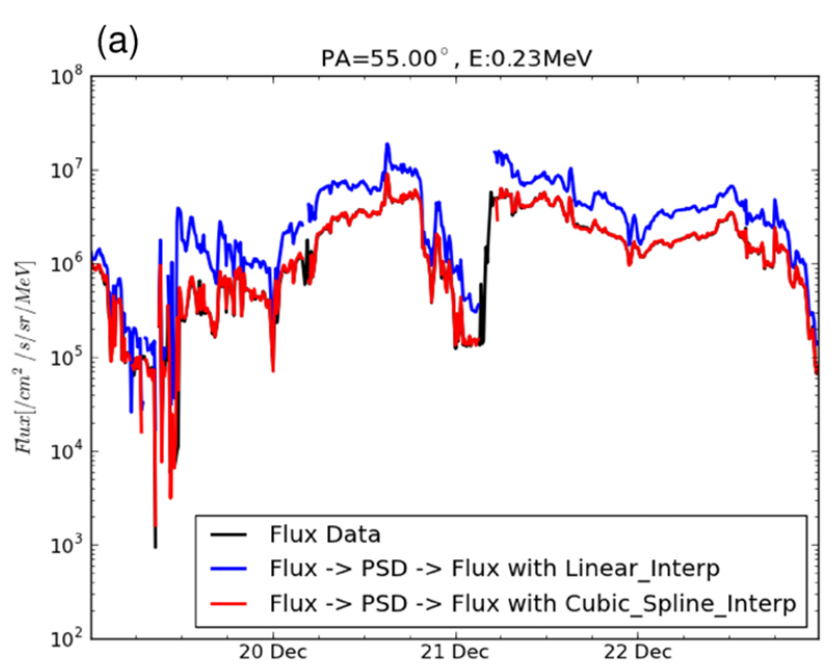

(b)

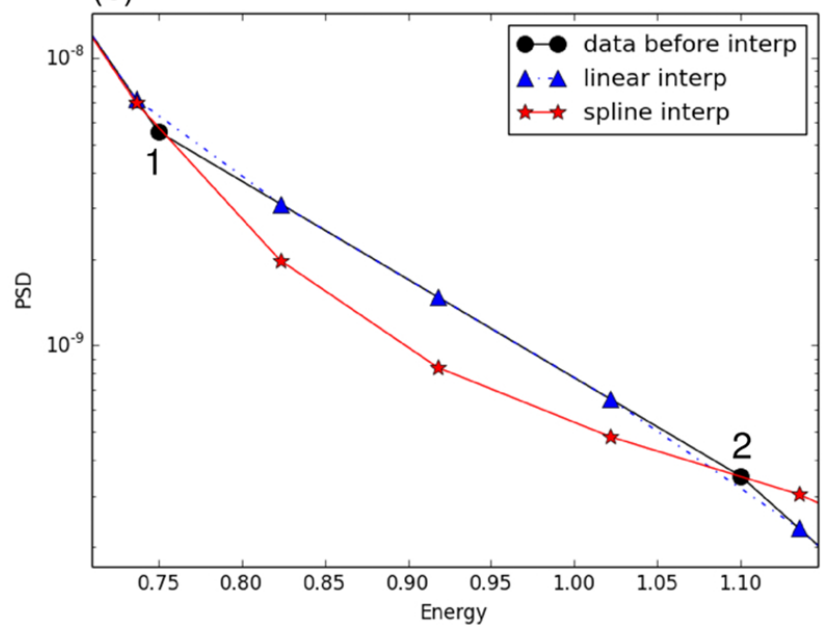

Fig. 1. (a) Comparisons between original flux data (black) and reversely inverted flux from the phase space density (PSD) information, which has been previously converted from the original flux data. The conversion between the flux and PSD utilizes a cubic spline interpolation (red) or a linear interpolation method (blue) within the energy spectrum. (b) The illustration of the two methods in interpolating the PSD over an energy spectrum. When the reverse inversion is converted, the newly interpolated data at point 1 using the linear method is above the original black dot (on the dash line). This is however the opposite at point 2 when the energy spectrum has a convex shape.

Koller, 2012). In this study, we use the EnKF implemented with a LAI technique to properly account for model errors.

First, we briefly describe the general algorithm of the EnKF in this section following Evensen (2003). The EnKF combines the observations and physical model forecast, both containing noises and inaccuracies, and produces an optimal estimation of the state variable. In the radiation belt data assimilation, the "observation" usually refers to the PSD observations converted from flux measurements, while the "model forecast" refers to the PSD value output from the radial diffusion model (Eq. 1). The model state vector at time $t$, denoted by $\boldsymbol{x}^{\mathrm{f}}(t)$, includes the modeled PSD state at each grid point from $L^{*}=1$ to 10 with grid size of 0.1 , while the observation vector at time $t$, denoted by $\boldsymbol{y}^{0}(t)$, includes the PSD observations inverted from the flux data. The assimilated state $\mathbf{x}^{\mathrm{a}}(t)$ of an ensemble with $N$ members thereafter becomes

$$
\begin{aligned}
& \boldsymbol{x}_{i}^{\mathrm{a}}(t)=\boldsymbol{x}_{i}^{\mathrm{f}}(t)+\mathbf{K}(t) \boldsymbol{d}_{i}(t), i=1,2, \ldots, N \\
& \mathbf{K}(t)=\mathbf{P}^{\mathrm{f}}(t) \mathbf{H}^{T}(t)\left(\mathbf{H}(t) \mathbf{P}^{\mathrm{f}}(t) \mathbf{H}^{T}(t)+\mathbf{R}^{0}(t)\right)^{-1},
\end{aligned}
$$

where $\boldsymbol{d}_{i}(t)=\boldsymbol{y}_{i}^{0}(t)-\mathbf{H}(t) \boldsymbol{x}_{i}^{\mathrm{f}}(t)$ is the innovation vector, residual between the observations and the model forecast; $\mathbf{K}$ is the Kalman gain matrix or weight matrix; $\mathbf{P}^{\mathrm{f}}$ and $\mathbf{R}^{0}$ are the error covariance matrices of the model forecast and observations, respectively; $\mathbf{H}(t)$ is a linear operator (size $n \times m$, where $n$ is the number of observations at time $t$ and $m$ is the number of grids in the model) projecting the model state vector onto the observational space at each time step with mostly zeros or ones.

In the EnKF the forecast error covariance $\mathbf{P}^{\mathbf{f}}$ is approximated by using the ensemble of model forecast as

$\mathbf{P}^{\mathrm{f}}=\frac{1}{N-1} \sum_{i=1}^{N}\left(\boldsymbol{x}_{i}^{\mathrm{f}}-\overline{\boldsymbol{x}^{\mathrm{f}}}\right)\left(\boldsymbol{x}_{i}^{\mathrm{f}}-\overline{\boldsymbol{x}^{\mathrm{f}}}\right)^{T}$,

where $\overline{\boldsymbol{x}}$ is the average of the $N$ forecast ensemble. For our experiments, an ensemble of 50 model simulations using the 1-D radiation belt model is created by introducing random small perturbations to the initial state vector (Koller et al., 2007). These ensemble members are used to resemble the uncertainty in the model forecast. Each ensemble member is advanced individually and the error covariance matrix propagates with time.

The observational error covariance $\mathbf{R}^{0}$ is determined from random noises taken from a normal distribution with zero mean and a specified standard deviation of $30 \%$ based on a conjunction investigation of geosynchronous measurements (Koller et al., 2007).

$\mathbf{R}^{0}=\frac{1}{N-1} \sum_{i=1}^{N} \varepsilon_{i} \varepsilon_{i}^{T}$,
$\varepsilon_{i}=\boldsymbol{y}_{i}^{0^{\prime}}(t)-\boldsymbol{y}_{i}^{0}(t)$,

where $\boldsymbol{y}_{i}^{0^{\prime}}(t)$ is the perturbed observation.

Consequently, each ensemble member $\boldsymbol{x}_{i}^{\mathrm{a}}$ is advanced by the radiation belt model with a model operator $M$ via $\boldsymbol{x}_{i}^{\mathrm{f}}\left(t^{\prime}\right)=M \boldsymbol{x}_{i}^{\mathrm{a}}(t)$, and the new forecast ensemble is updated in the next assimilation cycle following Eq. (4). The average values of the forecast ensemble and the assimilated ensemble provide new forecast output and new reanalysis output from the data assimilation, respectively.

It should be noted that, although the spread of the ensemble members defines the uncertainty of the model forecast, 
it usually does not include adequate model errors caused by the neglected physics or numerical approximation. The insufficient representation of model errors can result in the underestimation of forecast uncertainty, leading to the filter divergence where the observations are completely ignored in the assimilation. To avoid this behavior, we use the LAI technique to locally inflate the forecast error covariance $\mathbf{P}^{\mathrm{f}}$ where observations are available, in order to appropriately compensate for possibly ill-considered model errors (see Godinez and Koller, 2012, for details). The assimilation is then performed using the inflated forecast error covariance $\mathbf{P}^{\mathbf{f}^{\prime}}$ to construct the analysis state.

\section{Data assimilation results}

With the above techniques and tools implemented, we carry out data assimilation for a moderate storm event on 1922 December 2002 using measurements from LANL-97A, LANL-90, and Polar spacecraft. Figure 2 shows the solar wind, interplanetary magnetic field (IMF), and geomagnetic conditions for this event. The southward IMF $B_{z}$ excursion lasted for a few hours on 19 December. The SYM-H index dropped to $-80 \mathrm{nT}$ during the storm main phase and then slowly recovered. Beginning in the late hours of 21 December, $B_{z}$ again stayed in the southward orientation for a long time with the SYM-H index decreasing to $-90 \mathrm{nT}$. A sudden solar wind dynamic pressure pulse then impacted the magnetosphere at 03:00 UT of 21 December when $B_{z}$ turned back to the northward direction, after which the system slowly recovered to a quiet state.

To estimate the potential improvement in the radiation belt data assimilation by using a physics-based magnetic field configuration, the results are compared with another assimilation experiment using an empirical magnetic field configuration (i.e., T01S). Figure 3 shows the assimilated radiation belt state with the PSD data obtained by converting flux measurements from LANL-GEO (LANL-97A and 1990-095) and Polar spacecraft, using the RAM-SCB (left) or T01S (right) magnetic field configuration. Both assimilated radiation belt states show similarities in the location of the PSD peak and the temporal evolution of the PSD, such as decrease in the main phase when $B_{z}$ turns southward (19 December 12:00-20 December), the PSD dropout when a solar wind dynamic pressure pulse arrives at the magnetopause (early 21 December), and the recovery of PSD in the outer region (22 December). These similarities suggest that the 1-D radiation belt data assimilation reproduces the main trends of radiation belt dynamics irrespective of the global field model complexity and accuracy, which is consistent with the conclusion in $\mathrm{Ni}$ et al. (2009).

However, the log-normalized difference between the two assimilated states (Fig. 3c)

$$
\frac{\log f_{\text {RAMSCB }}-\log f_{\text {T01S }}}{0.5\left(\log f_{\text {RAMSCB }}+\log f_{\text {T01S }}\right)_{\text {max }}}
$$

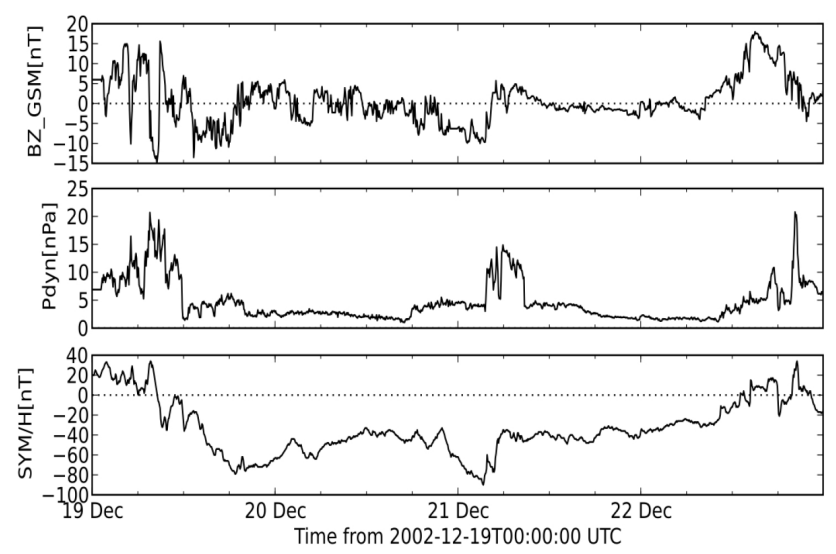

Fig. 2. The solar wind interplanetary and geomagnetospheric conditions from 19 December to 22 December 2012.

reveals that there is notable discrepancy between the two, particularly in the extent to which the data assimilation reproduces the PSD decrease during the main phase and the PSD increase in the recovery phase. The blue color in the storm main phase indicates a more intense decrease in the PSD when using the RAM-SCB magnetic field configuration, while the red in the recovery phase means that the assimilated PSD experiences more intensification. These disparities strongly imply that, even though various magnetic field models may reproduce qualitatively the dynamic radiation belt features, the choice of a global magnetic field model used for the PSD data conversion can influence significantly the assimilated magnitude of the radiation belt electron content and is important for the accurate quantitative representation of the radiation belt.

Given the discrepancy between the two assimilation experiments, the question of which assimilation experiment produces a more realistic radiation belt is of great practical importance to the forecasting of the hazardous radiation environment. There are two ways to assess the accuracy of the assimilation experiment: (1) which magnetic field model is more accurate, and (2) which assimilative PSD prediction is more realistic. For the first one, since the boundary condition for the RAM-SCB model is taken from the Kp-driven T89 model, it may not fully reflect the dynamics associated with largely disturbed solar wind dynamic pressure. Therefore, the prediction of global configuration with RAM-SCB may not be as accurate during the storm main phase, and the global magnetosphere may not be as stretched as in reality. As a result, the computed adiabatic values $\left(\mu, K, L^{*}\right)$ and PSD data can be underestimated. This may explain the extensive reduction of PSD during the storm main phase using the RAM-SCB model.

The second one can be assessed through comparisons with independent observations (i.e., out-of-sample data for the assimilation), as described below. Such a comparison will in turn suggest the fidelity of a global magnetic field model. 
(a) RAM-SCB
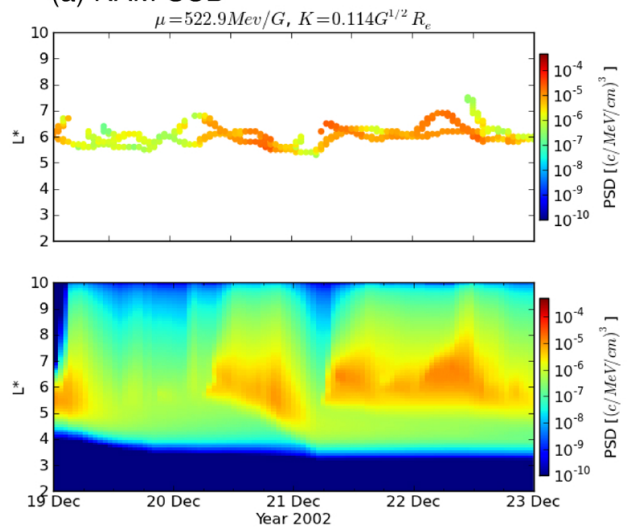

(b) T01Storm
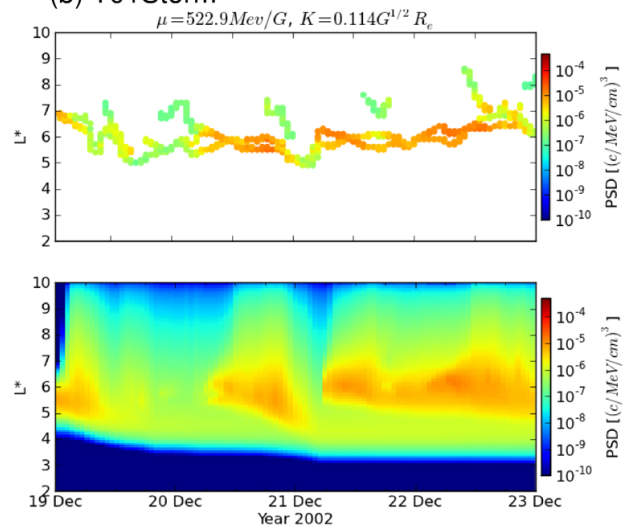

(c) normalized difference

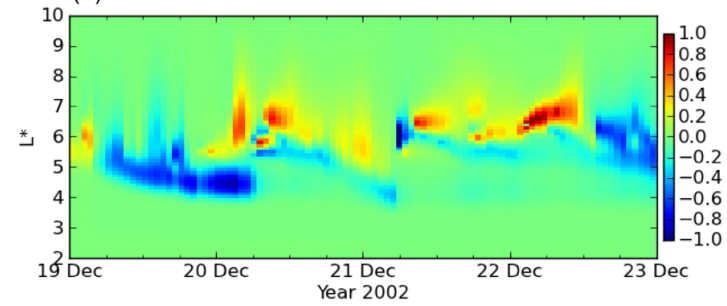

Fig. 3. (a) Top panel shows the PSD $\left(L^{*}\right)$ data converted from flux observations measured by LANL-97A, LANL-90, and Polar spacecraft by means of a global magnetic field configuration generated by the RAM-SCB model. The $\mu$ is $522.9 \mathrm{Mev} \mathrm{G}^{-1}$ and $K$ is $0.114 G^{1 / 2} R_{\mathrm{E}}$. Bottom panel shows the assimilated PSD in the outer electron electron radiation belt. (b) shows the PSD $\left(L^{*}\right)$ data and the assimilated PSD based on the T01S magnetic field model. (c) shows the normalized difference between the two assimilated radiation belt states. The red indicates that the assimilated PSD is larger when using the RAM-SCB magnetic field configuration, while the blue means the opposite.

This is because the accuracy of the PSD data converted from the flux observation is subject to the perfection of a magnetic field model. A more realistic assimilation result based on the $\operatorname{PSD}\left(L^{*}\right)$ data will suggest a more realistic global magnetic field configuration. That is, a better flux prediction from the assimilation experiment can indicate that the selected magnetic field model represents a more realistic global magnetosphere.

\subsection{PSD prediction by assimilation}

Verifying the accuracy of the data assimilation prediction is limited because we cannot observe PSD directly. Despite of this fact, the performance of the data assimilation can be verified using the PSD data from an independent satellite. Figure 4 exhibits the PSD comparison from the assimilation state ( $y$ axis) and from the observation ( $x$ axis) used in the data assimilation (panels a, b, d, e) or at an out-of-sample satellite, LANL 1991-080 (panels c, f). The top row is from the assimilation using the RAM-SCB magnetic field configuration, and the bottom one uses the T01S magnetic fields. Various energy levels $(\mu)$ are shown with $K \simeq 0.1 G^{1 / 2} R_{\mathrm{E}}$. While the assimilated PSD generally preserves the input PSD data (see the high cross-correlation coefficient and small normalized root-mean-square (nRMS) error in panels a, b, d, and e), the reconstructed PSD for an independent satellite also shows good agreement with observations in panels $\mathrm{c}$ and $\mathrm{f}$. This is an indication of a good reanalysis of the radiation belt with data from two LANL-GEO satellites and one Polar spacecraft.

\subsection{Flux prediction by assimilation}

To better evaluate the accuracy of the data assimilation in predicting the realistic radiation belt, we utilize the original flux observation. The assimilation is believed to provide an accurate estimation of the realistic radiation environment near the location of the satellite if the assimilated results predict a flux that matches the observations from an independent satellite. Figure 5 displays flux comparisons between the prediction from the assimilation (green dots) and the observation (blue lines) at three LANL-GEO satellites. Fluxes at two energy levels $(0.225 \mathrm{MeV}, 1.1 \mathrm{MeV})$ with pitch angle of $55^{\circ}$ are shown in each panel. The predicted flux is inferred from the assimilated PSD following the reversed steps as outlined in Sect. 2.2.2.

Along two satellite trajectories (LANL-97A, LANL-90), the predicted flux from the data assimilation agrees well with the observed flux for both energy levels. This is expected since these satellites provide the input PSD data for the data 

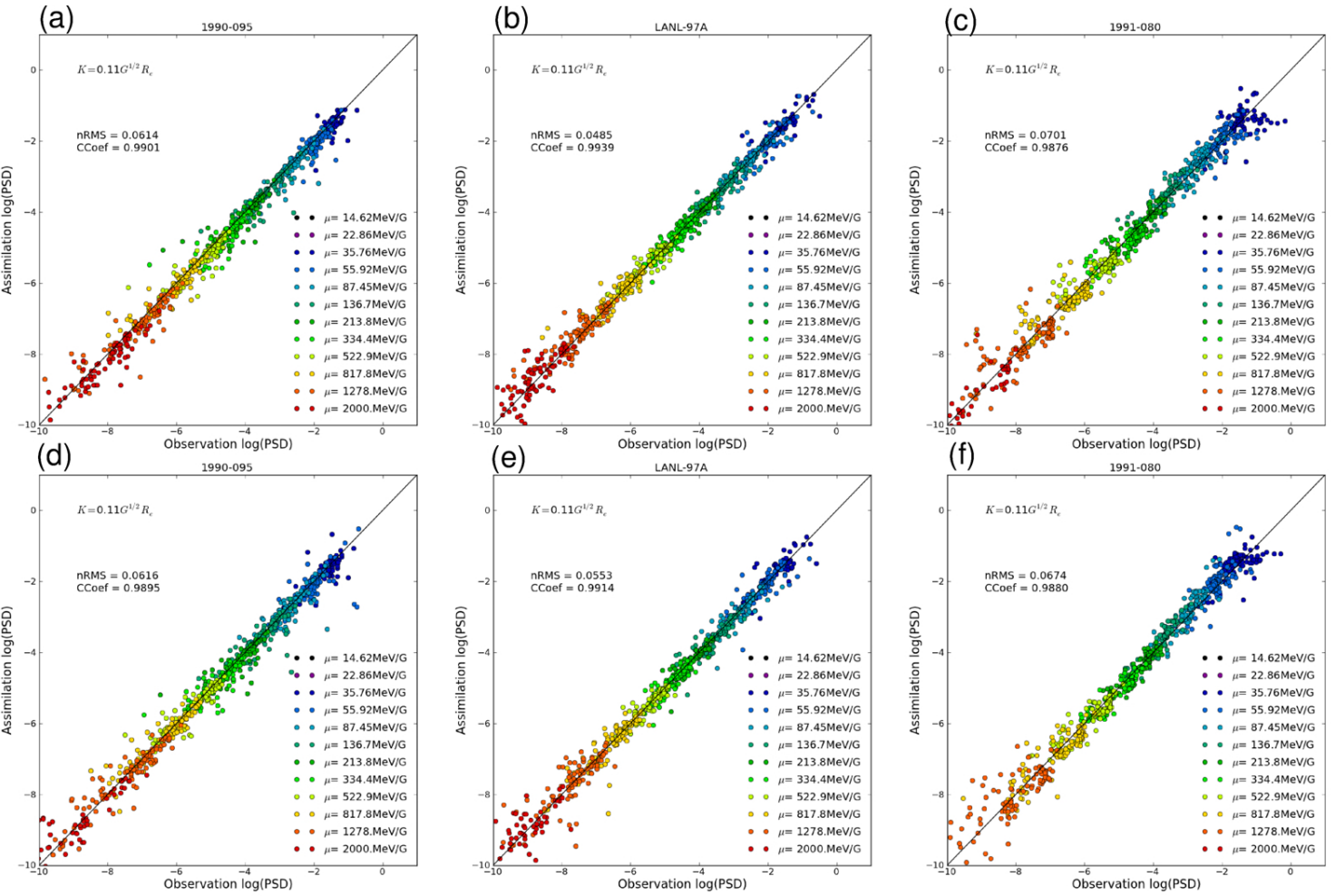

Fig. 4. The assimilated PSD ( $y$ axis) and the observed PSD ( $x$ axis) for various $\mu$ values and $K \simeq 0.1 G^{1 / 2} R_{\mathrm{E}}$. The observed PSD for the first two columns is obtained from two satellites (1990-095, LANL-97A), used as input in the assimilation. The PSD data on the right column are from an independent satellite (1991-080) that does not participate in the assimilation. The top row is for the assimilation using the RAM-SCB magnetic fields, and the bottom row is for the assimilation using T01S magnetic fields. The normalized root-mean-square (nRMS) error and the cross-correlation coefficient are denoted in each panel.

assimilation. This further proves that the data assimilation successfully takes into account the observations.

On the other hand, the comparison between the predicted flux and available measurement along an independent geostationary satellite trajectory is more meaningful in evaluating the capability of data assimilation in reproducing the realistic radiation belt state. We choose an independent satellite, 1991-080, for such a comparison. Bottom panels in Fig. 5 show that the flux predicted by both assimilations agrees reasonably well with the observed flux. The predicted flux from the data assimilation using the RAM-SCB magnetic field configuration shows a better agreement with the lowenergy flux observation, resulting in a smaller nRMS error and a larger cross-correlation coefficient. However the highenergy flux is predicted slightly worse than that using the T01S magnetic fields.

The above flux comparisons indicate a reasonably good prediction during the whole storm period. However, given that the two data assimilation experiments in Fig. 4c show distinct difference during different storm phases, next we examine the flux prediction separately during the storm main phase and recovery phase. The storm main phase is picked from 19 December 08:00 UT through 20 December 00:00 UT and from 21 December 00:00 UT through 21 December 06:00 UT, during which the radiation belt electron PSD is depleted. The recovery phase is picked from 20 December 00:00 UT through 21 December 00:00 UT and from 21 December 06:00 UT through 23 December 00:00 UT, during which the electron PSD is enhanced. Table 1 lists the nRMS errors for the flux agreement at different energy levels and pitch angle of $55^{\circ}$. All these nRMS errors are very small. During the main phase, the data assimilation using the T01S magnetic field configuration shows a better prediction of the flux, while during the recovery phase the one using the RAMSCB magnetic field configuration shows a better flux prediction for most of the energy levels. This may be attributed to the model parameter at the boundary. Since the RAM-SCB uses the T89 model to provide the magnetic field boundary, it can not fully capture the solar interplanetary dynamics as the T89 is merely Kp dependent. Thus, during the main phase when the solar interplanetary structures are disturbed, the RAM-SCB could not represent the global magnetosphere so well. In contrast, during the recovery phase when the solar wind conditions are relatively quiet, the RAM-SCB can represent the global configuration well and even better than that by the T01S. To conclude, with a different global magnetic 
(a) RAM-SCB

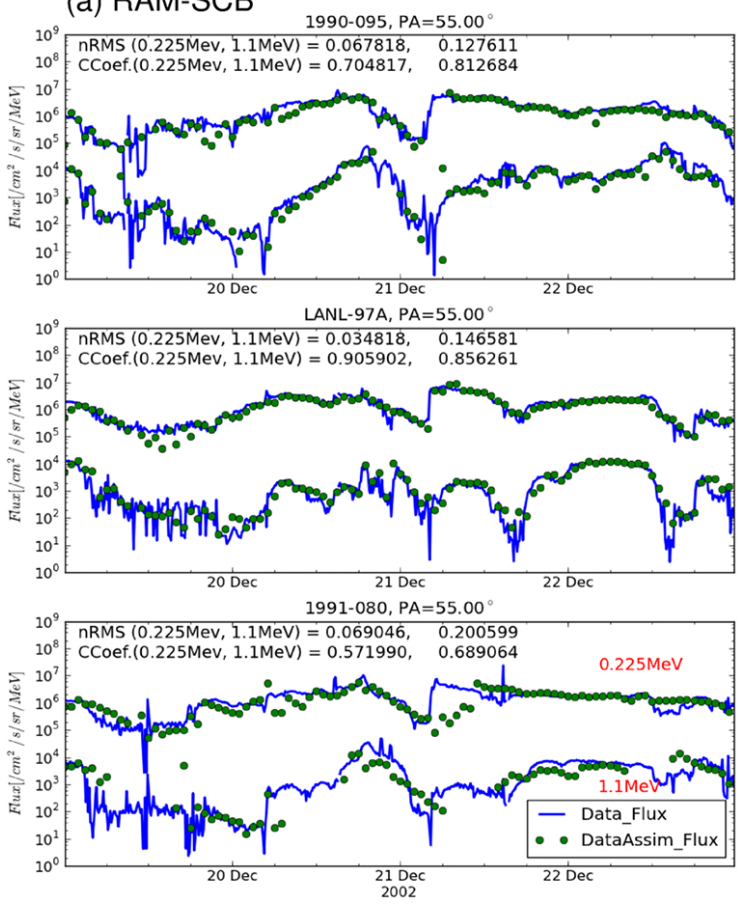

(b) T01S
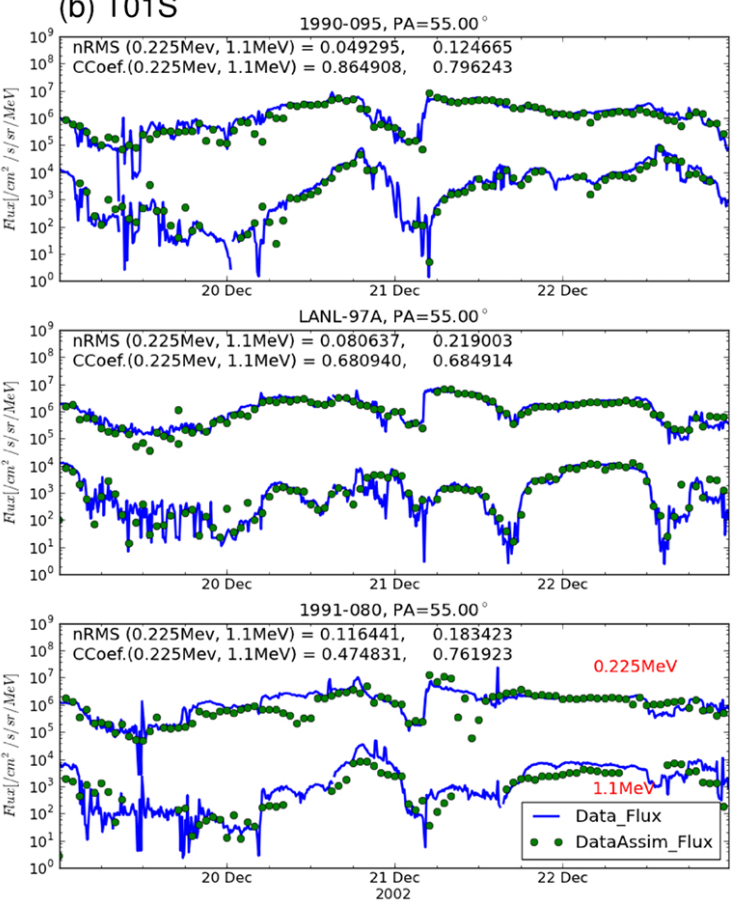

Fig. 5. The predicted flux (green) from the assimilated radiation environment along three LANL-GEO satellite trajectories, compared to original flux observations (blue). The nRMS error and cross-correlation coefficients are denoted for each comparison. The top two rows are along the satellites that provided the input PSD data to the data assimilation, while the bottom row is from an independent trajectory. The left column shows the flux comparison from the data assimilation using the RAM-SCB magnetic field configuration, and the right column is the comparison from the data assimilation using the T01S magnetic field model.

Table 1. The normalized root-mean-square (nRMS) errors for the flux comparison during the main phase and recovery phase. A smaller nRMS error means a better flux prediction from the data assimilation.

\begin{tabular}{lrrr}
\hline Model & $\begin{array}{r}\text { Energy } \\
(\mathrm{MeV})\end{array}$ & $\begin{array}{r}\text { Main phase } \\
\text { nRMS }\end{array}$ & $\begin{array}{r}\text { Recovery } \\
\text { phase nRMS }\end{array}$ \\
\hline RAM-SCB & 0.105 & 0.0774 & 0.0530 \\
& 0.225 & 0.1016 & 0.0593 \\
& 0.50 & 0.1174 & 0.0867 \\
& 1.10 & 0.2731 & 0.1458 \\
\hline T01S & 0.105 & 0.0755 & 0.0591 \\
& 0.225 & 0.0666 & 0.0653 \\
& 0.50 & 0.0806 & 0.1017 \\
& 1.10 & 0.2200 & 0.1412 \\
\hline
\end{tabular}

field model, the radiation belt data assimilation similarly produces the temporal evolution of the radiation belt, but it influences the accuracy of the radiation environment in different storm phases, depending on the model capability in representing the global magnetosphere configuration during those phases.

It should be noted that as the nRMS error aggregates various samples in the chosen time interval into one single measure to evaluate the average agreement, it would be expected that some local samples demonstrate different implications than the average agreement. For example, the agreement using RAM-SCB appears worse than using T01S in the early recovery phase around 21 December 06:00 UT. This is contrary to what the nRMS error suggests in Table 1. However, we are more interested in discovering the general agreement for a longer time period. It should also be noted that the phase-dependent performance is probably subject to storm events. In the future, we will study more storm periods to further identify the difference between using a complex physicsbased model and using empirical magnetic field models.

\section{Summary}

This study carried out a radiation belt data assimilation based on a 1-D radial diffusion model and electron phase space density data obtained from LANL-GEO satellites and Polar spacecraft. The PSD data were converted from flux observations with the adiabatic coordinates $\left(\mu, K, L^{*}\right)$ computed based on a global magnetic field configuration generated by the physics-based magnetically self-consistent inner magnetosphere model RAM-SCB. The conversion was carried out by using a cubic spline interpolation method, rather than a linear interpolation within the energy spectrum. The former 
method allowed for a more accurate flux-to-PSD conversion by eliminating unnecessary bias which appears when using the latter method. A recently developed localized adaptive inflation technique was used in the ensemble Kalman filter to appropriately account for model errors, successfully reconstructing the radiation belt dynamics. We conducted another data assimilation using PSD data converted based on the magnetic field configuration from the empirical T01S model in order to examine the dependence on the selection of a global magnetic field model.

Results indicated that both data assimilations captured similar temporal evolution in the outer electron radiation belt as well as the location of the PSD peak, the PSD dropout during the storm main phase, and energization in the recovery phase. Nevertheless, the magnitude of the PSD decrease and energization in these phases was more intense in the case using RAM-SCB magnetic field configuration. We further compared the assimilated radiation belt state with observations along an independent LANL-GEO satellite trajectory to examine the accuracy of the assimilated radiation environment. Both data assimilations demonstrated good prediction of both PSD and flux at the independent satellite. A closer comparison in different storm phases suggests that for the chosen storm event the RAM-SCB model produces a better global configuration during the storm recovery phase and therefore a better reconstruction of radiation belt through the data assimilation than the T01S model, but it behaves slightly worse during the storm main phase due to the deficiency on the boundary specification.

Acknowledgements. This work was conducted under the auspices of the US Department of Energy, with support from the Los Alamos National Laboratory Directed Research and Development program. The authors thank Yue Chen and Geoff Reeves for helpful discussion.

Topical Editor L. Blomberg thanks two anonymous referees for their help in evaluating this paper.

\section{References}

Bourdarie, S., Friedel, R. H. W., Fennell, J., Kanekal, S., and Cayton, T. E.: Radiation belt representation of the energetic electron environment: Model and data synthesis using the Salammbô radiation belt transport code and Los Alamos geosynchronous and GPS energetic particle data, Space Weather, 3, S04S01, doi:10.1029/2004SW000065, 2005.

Bourdarie, S. A. and Maget, V. F.: Electron radiation belt data assimilation with an ensemble Kalman filter relying on the Salammbô code, Ann. Geophys., 30, 929-943, doi:10.5194/angeo-30-929-2012, 2012.

Brautigam, D. H. and Albert, J. M.: Radial diffusion analysis of outer radiation belt electrons during the October 9, 1990, magnetic storm, J. Geophys. Res.-Space, 105, 291-310, doi:10.1029/1999JA900344, 2000.
Carpenter, D. L. and Anderson, R. R.: An ISEE/Whistler model of equatorial electron density in the magnetosphere, J. Geophys. Res.-Space, 97, 1097-1108, doi:10.1029/91JA01548, 1992.

Chen, Y., Friedel, R. H. W., Reeves, G. D., Onsager, T. G., and Thomsen, M. F.: Multisatellite determination of the relativistic electron phase space density at geosynchronous orbit: Methodology and results during geomagnetically quiet times, J. Geophys. Res.-Space, 110, A10210, doi:10.1029/2004JA010895, 2005.

Daae, M., Shprits, Y. Y., Ni, B., Koller, J., Kondrashov, D., and Chen, Y.: Reanalysis of radiation belt electron phase space density using various boundary conditions and loss models, Adv. Space Res., 48, 1327-1334, doi:10.1016/j.asr.2011.07.001, 2011.

Evensen, G.: The Ensemble Kalman Filter: theoretical formulation and practical implementation, Ocean Dynam., 53, 343-367, doi:10.1007/s10236-003-0036-9, 2003.

Godinez, H. C. and Koller, J.: Localized adaptive inflation in ensemble data assimilation for a radiation belt model, Space Weather, 10, S08001, doi:10.1029/2012SW000767, 2012.

Huang, C.-L., Spence, H. E., Singer, H. J., and Tsyganenko, N. A.: A quantitative assessment of empirical magnetic field models at geosynchronous orbit during magnetic storms, J. Geophys. Res.Space, 113, A04208, doi:10.1029/2007JA012623, 2008.

Jordanova, V. K., Kozyra, J. U., Khazanov, G. V., Nagy, A. F., Rasmussen, C. E., and Fok, M.-C.: A bounce-averaged kinetic model of the ring current ion population, Geophys. Res. Lett., 21, 27852788, doi:10.1029/94GL02695, 1994.

Jordanova, V. K., Miyoshi, Y. S., Zaharia, S., Thomsen, M. F., Reeves, G. D., Evans, D. S., Mouikis, C. G., and Fennell, J. F.: Kinetic simulations of ring current evolution during the Geospace Environment Modeling challenge events, J. Geophys. Res.-Space., 111, A11S10, doi:10.1029/2006JA011644, 2006.

Jordanova, V. K., Zaharia, S., and Welling, D. T.: Comparative study of ring current development using empirical, dipolar, and selfconsistent magnetic field simulations, J. Geophys. Res.-Space, 115, A00J11, doi:10.1029/2010JA015671, 2010.

Jordanova, V. K., Yu, Y., Niehof, J. T., Skoug, R. M., Reeves, G. D., Kletzing, C. A., Fennell, J. F., and Spence, H. E.: Simulations of inner magnetosphere dynamics with an expanded RAM-SCB model and comparisons with Van Allen Probes observations, Geophys. Res. Lett., online first, doi:10.1002/2014GL059533, 2014.

Koller, J., Chen, Y., Reeves, G. D., Friedel, R. H. W., Cayton, T. E., and Vrugt, J. A.: Identifying the radiation belt source region by data assimilation, J. Geophys. Res.-Space, 112, A06244, doi:10.1029/2006JA012196, 2007.

Kondrashov, D., Shprits, Y., Ghil, M., and Thorne, R.: A Kalman filter technique to estimate relativistic electron lifetimes in the outer radiation belt, J. Geophys. Res.-Space, 112, A10227, doi:10.1029/2007JA012583, 2007.

Kondrashov, D., Ghil, M., and Shprits, Y.: Lognormal Kalman filter for assimilating phase space density data in the radiation belts, Space Weather, 9, S11006, doi:10.1029/2011SW000726, 2011.

Morley, S. K., Welling, D. T., Koller, J., Larsen, B. A., Henderson, M. G., and Niehof, J.: Spacepy - a python-based library of tools for the space sciences, in: Proceedings of the 9th Python in Science Conference, edited by: van der Walt, S. and Millman, J., 39-45, 2010. 
Naehr, S. M. and Toffoletto, F. R.: Radiation belt data assimilation with an extended Kalman filter, Space Weather, 3, S06001, doi:10.1029/2004SW000121, 2005.

Ni, B., Shprits, Y., Thorne, R., Friedel, R., and Nagai, T.: Reanalysis of relativistic radiation belt electron phase space density using multisatellite observations: Sensitivity to empirical magnetic field models, J. Geophys. Res.-Space, 114, A12208, doi:10.1029/2009JA014438, 2009.

Ni, B., Shprits, Y., Hartinger, V. Angelopoulos, Gu, X., and Larson, D.: Analysis of radiation belt energetic electron phase space density using THEMIS SST measurements: Cross-satellite calibration and a case study, J. Geophys. Res.-Space, 116, A03208, doi:10.1029/2010JA016104, 2011.

Roederer, J. G.: Dynamics of geomagnetically trapped radiation, Physics and Chemistry in Space, Berlin: Springer, 1970.

Schiller, Q., Li, X., Koller, J., Godinez, H., and Turner, D. L.: A parametric study of the source rate for outer radiation belt electrons using a Kalman filter, J. Geophys. Res.-Space, 117, A09211, doi:10.1029/2012JA017779, 2012.

Shprits, Y. Y., Thorne, R. M., Friedel, R., Reeves, G. D., Fennell, J., Baker, D. N., and Kanekal, S. G.: Outward radial diffusion driven by losses at magnetopause, J. Geophys. Res.-Space, 111, A11214, doi:10.1029/2006JA011657, 2006.

Shprits, Y., Kondrashov, D., Chen, Y., Thorne, R., Ghil, M., Friedel, R., and Reeves, G.: Reanalysis of relativistic radiation belt electron fluxes using CRRES satellite data, a radial diffusion model, and a Kalman filter, J. Geophys. Res.-Space, 112, A12216, doi:10.1029/2007JA012579, 2007.

Shprits, Y., Daae, M., and Ni, B.: Statistical analysis of phase space density buildups and dropouts, J. Geophys. Res.-Space, 117, A01219, doi:10.1029/2011JA016939, 2012.

Shprits, Y. Y., Kellerman, A., Kondrashov, D., and Subbotin, D.: Application of a new data operator-splitting data assimilation technique to the 3-D VERB diffusion code and CRRES measurements, Geophys. Res. Lett., 40, 4998-5002, doi:10.1002/grl.50969, 2013.

Tsyganenko, N. A.: A magnetospheric magnetic field model with a warped tail current sheet, Planetary Space Sci., 37, 5-20, doi:10.1016/0032-0633(89)90066-4, 1989.
Tsyganenko, N. A. and Mukai, T.: Tail plasma sheet models derived from Geotail particle data, J. Geophys. Res.-Space, 108, 1136, doi:10.1029/2002JA009707, 2003.

Weimer, D. R.: An improved model of ionospheric electric potentials including substorm perturbations and application to the Geospace Environment Modeling November 24, 1996, event, J. Geophys. Res.-Space, 106, 407-416, doi:10.1029/2000JA000604, 2001.

Welling, D. T., Koller, J., and Camporeale, E.: Verification of SpacePy's radial diffusion radiation belt model, Geosci. Model Dev., 5, 277-287, doi:10.5194/gmd-5-277-2012, 2012.

Yu, Y., Jordanova, V., Zaharia, S., Koller, J., Zhang, J., and Kistler, L. M.: Validation study of the magnetically self-consistent inner magnetosphere model RAM-SCB, J. Geophys. Res.-Space, 117, A03222, doi:10.1029/2011JA017321, 2012a.

Yu, Y., Koller, J., Zaharia, S., and Jordanova, V.: $L^{*}$ neural networks from different magnetic field models and their applicability, Space Weather, 10, S02014, doi:10.1029/2011SW000743, 2012b.

Yu, Y., Koller, J., Jordanova, V., Zaharia, S., Friedel, R., Morley, S., Chen, Y., Baker, D., Reeves, G., and Spence, H.: Applicatin and testing of the $L^{*}$ neural network with the self-consistent magnetic field model of RAM-SCB, J. Geophys. Res.-Space, online first, doi:10.1002/2013JA019350, 2014.

Zaharia, S.: Improved Euler potential method for three-dimensional magnetospheric equilibrium, J. Geophys. Res.-Space, 113, A08221, doi:10.1029/2008JA013325, 2008.

Zaharia, S., Cheng, C. Z., and Maezawa, K.: 3-D force-balanced magnetospheric configurations, Ann. Geophys., 22, 251-265, doi:10.5194/angeo-22-251-2004, 2004.

Zaharia, S., Jordanova, V. K., Thomsen, M. F., and Reeves, G. D.: Self-consistent modeling of magnetic fields and plasmas in the inner magnetosphere: Application to a geomagnetic storm, J. Geophys. Res.-Space, 111, A11S14, doi:10.1029/2006JA011619, 2006. 